\title{
Research on the Forming Mechanism of Micro/ Nano Features during the Cast Molding Process
}

\author{
Xiangdong Ye $\mathrm{Y}^{1,2}$, Yugang Duan ${ }^{2}$, Yucheng Ding ${ }^{2, *}$
}

(Received 12 October 2011; accepted 08 December 2011; published online 23 December 2011.)

\begin{abstract}
Cast molding process has provided a reliable, simple and cost-effective way to fabricate micro structures since decades ago. In order to obtain structures with fine, dense and deep nano-size features by cast molding, it is necessary to study the forming mechanism in the process. In this paper, based on major steps of cast molding, filling models of liquid are established and solved; and the forming mechanism of liquid is revealed. Moreover, the scale effect between the liquid and the cavity on the filling velocity of liquid is studied. It is also interesting to find out that the wettability of liquid on the cavity may be changed from wetting to dewetting depends on the pressure difference. Finally, we experimentally verify some of our modeling results on the flowing and filling state of the liquid during the cast molding process.
\end{abstract}

Keywords: Forming mechanism; Cast molding process; Nano-size features; Liquid

Citation: Xiangdong Ye, Yugang Duan and Yucheng Ding, "Research on the Forming Mechanism of Micro/Nano Features during the Cast Molding Process", Nano-Micro Lett. 3 (4), 249-255 (2011). http://dx. doi.org/10.3786/nml.v3i4.p249-255

\section{Introduction}

Two decades ago, cast molding had been used to fabricate microstructures in fluid integrated circuit [1]. Up to now, many researchers have utilized the technique to fabricate various micro/nano structures. The utilization of cast molding can be roughly classified into two main strategies. One is to make the final structures by the technique directly, such as fabrication of three dimensional microfluidic systems [2], devices integration with large-area nanopillar arrays [3], double casting of microstructured masters [4], and optical switching of liquid crystal grating [5]. Another is to make templates by the technique firstly, and then the templates are used to fabricate the final structures. The second approach has been widely developed since imprint lithography was proposed, such as soft lithography [6] and UV imprint lithography [7-9]. By the use of fabricated templates, many complicated micro/nano structures can be replicated in a large variety of materials and in large area efficiently, such as polymethylmethacrylate, ceramics, polystyrene, polyurethane and Teflon [10-15].

In the two above-mentioned approaches, the cast molding process provides a reliable, simple and costeffective way to construct structures for many materials. However, it is still a challenge to obtain highquality structures with fine, deep, and compact nanosize features by cast molding process. In 1999, Chiang et al. [16] investigated castable materials and mold sticking properties, resolution of pattern transfer, and effects of surface roughness and surface wettability. In our previous work, effects of vacuum degree and the curing temperature on the quality of PDMS mold were studied; and the process was optimized to obtain PDMS molds with high fidelity and improved physical properties [17].

Cast molding process can usually be divided into four

\footnotetext{
${ }^{1}$ Key Laboratory of Photonic and Electronic Materials, School of Electronic Science and Engineering, Nanjing University, Nanjing 210093, China

${ }^{2}$ State Key Laboratory of Manufacturing Systems Engineering, Xi'an Jiaotong University, Xi'an 710049, China

*Corresponding author. E-mail: ycding@xjtu.edu.cn
} 
steps: Firstly, the liquid is poured onto the surface of a master under atmospheric environment. Secondly, the assembly is evacuated to eliminate the bubbles. Then the assembly is taken out to return to the atmospheric environment again. Finally, the liquid is cured under a specific condition. The first three steps can be grouped into the forming stage, and the last step can be named as the curing stage. During the first stage, the liquid flows along the surface of the master and penetrates the grooves in the master gradually, and forms the micro/nano features in the liquid. Thus, the first stage can be called the forming stage, which is the basis of constructing the structures in the liquid and will affect the fidelity of the replicated structures directly.

Therefore, it is essential to analyze the effects of vacuum degree, liquid viscosity, surface tension, contact angle and scale effect on the flowing and penetrating state of the liquid during the forming stage. In this paper, by establishing and solving the flowing and filling model of the liquid during the forming stage, the effects of the above factors are analyzed, and the forming mechanism is revealed. Finally, our experimental results on cast molding agree well with the above analysis.

\section{Analysis}

As the forming stage in cast molding process consists of three different steps, we will analyze the forming stage according to these steps respectively.

\section{Liquid penetrating into master under atmo- sphere}

If the liquid can wet the surface of the cavity (diameter $2 \mathrm{R}$, height $\mathrm{h}$ ) in master, the poured liquid will penetrate into the upper part of the cavity (height $h_{i}$ ) due to surface tension $\gamma$, as shown in the following Fig. 1,

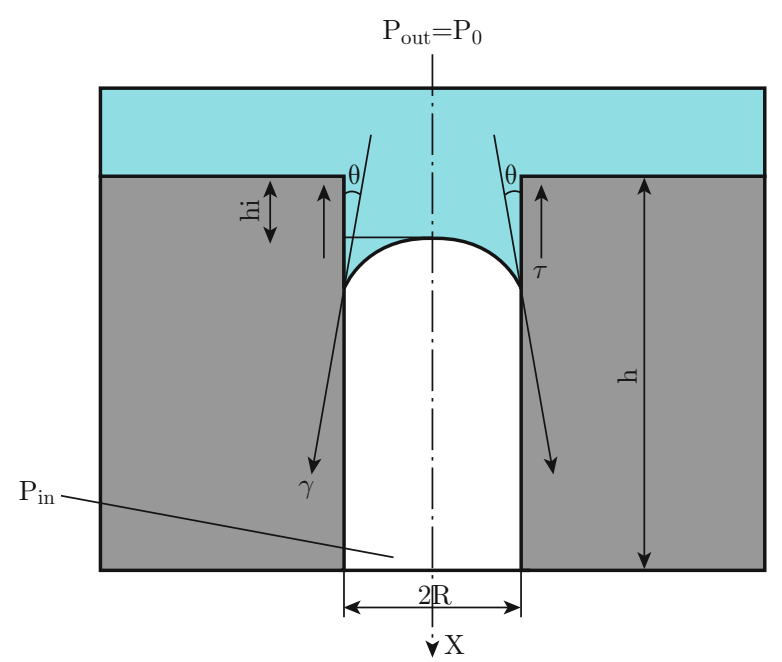

Fig. 1 The liquid cast over the cavity under atmosphere. contact angle of the liquid over the cavity wall is $\theta$, and air pressure in and out of the cavity are $\mathrm{P}_{\text {in }}$ and $\mathrm{P}_{\text {out }}\left(\mathrm{P}_{0}\right)$ respectively.

Suppose the liquid is incompressible, and the width of cavity is small, the liquid flowing into the cavity is stationary and laminar flow. We select an infinitesimal element (micro cylinder: radius $r$, height $h_{i}$ ) in the flowing liquid for further analysis, as shown in Fig. 2.

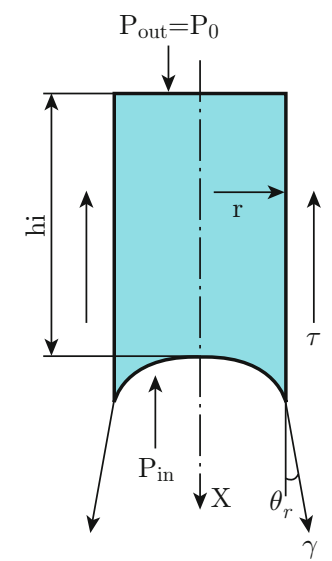

Fig. 2 State diagram of the infinitesimal element of the liquid under forces.

The infinitesimal element will be in equilibrium along axis $\mathrm{X}$ under actions of surface tension, internal friction $\tau$ and pressures, therefore:

$$
P_{0}\left(\pi r^{2}\right)-P_{i n}\left(\pi r^{2}\right)+\gamma \cos \theta_{r}(2 \pi r)-\tau\left(2 \pi r h_{i}\right)=0
$$

In the formula, the angle between $\gamma$ and axis $\mathrm{X}$ is $\theta_{r}$.

For liquid flowing in macroscopic scale cavities, Navier-Stokes Equation can work very well. However, if the cavities decrease to the nano scale, the continuum hypothesis which is the basis of Navier-Stokes Equation could become invalid. Some researchers have found that, for fluid flow in cavities larger than 10 times of fluid molecular diameters, the continuum hypothesis will still be valid and Navier-Stokes Equation can be used to analyze the flow $[18,19]$. However, as the fluid is confined in nano cavities, the fluid viscosity may be different from its bulk viscosity. Eringen et al. [20] proposed effective viscosity, which is the correction of bulk viscosity, depends on the fluid materials and the width of cavities:

$$
\mu=\mu_{b}\left[1+\xi\left(R_{g} / D\right)^{2}\right]
$$

where $\mu$ is the effective viscosity, $R_{g}$ is the radius of rotation of fluid molecular, $D$ is the external characteristic length, $\xi$ is a dimensionless constant, and $\mu_{b}$ is the bulk viscosity of fluid.

Therefore, according to internal friction $\tau=-\mu \frac{d v}{d r}(v$ is velocity of liquid along axis X) and Eq. (2), we can 
change Eq. (1) into following form:

$$
\begin{aligned}
& P_{0}\left(\pi r^{2}\right)-P_{i n}\left(\pi r^{2}\right)+\gamma \frac{r \cos \theta}{R}(2 \pi r)+ \\
& \mu_{b}\left[1+\xi\left(\frac{R_{g}}{R-r}\right)^{2}\right]\left(2 \pi r h_{i}\right) \frac{d v}{d r}=0 \\
& d v=-\frac{\left(P_{0}+\frac{2 \gamma \cos \theta}{R}-P_{i n}\right)}{2 \mu_{b} h_{i}} \times \\
& {\left[1-\frac{\xi R_{g}^{2}}{\xi R_{g}^{2}+(R-r)^{2}}\right] r d r }
\end{aligned}
$$

Integrating the Eq. (4) and substituting the boundary condition $(\nu(r=R)=0)$, we can obtain the velocity along the radius of the cavity as the following:

$$
\begin{aligned}
v= & \frac{\frac{2 \gamma \cos \theta}{R}-P_{0} \frac{h_{i}}{h-h_{i}}}{2 \mu_{b} h_{i}}\left\{\frac{1}{2}\left(R^{2}-r^{2}\right)+\frac{\xi R_{g}^{2}}{2} \ln [1+\right. \\
& \left.\left.\frac{(R-r)^{2}}{\xi R_{g}^{2}}\right]-\sqrt{\xi} R R_{g} \arctan \frac{(R-r)}{\sqrt{\xi} R_{g}}\right\}
\end{aligned}
$$

The maximum of the velocity is:

$$
\begin{aligned}
v_{\max }= & \frac{\frac{2 \gamma \cos \theta}{R}-P_{0} \frac{h_{i}}{h-h_{i}}}{2 \mu_{b} h_{i}}\left\{\frac{1}{2} R^{2}+\frac{\xi R_{g}^{2}}{2} \ln [1+\right. \\
& \left.\left.\frac{R^{2}}{\xi R_{g}^{2}}\right]-\sqrt{\xi} R R_{g} \arctan \frac{R}{\sqrt{\xi} R_{g}}\right\}
\end{aligned}
$$

If using $B$ as the ratio of cavity width and liquid radius of rotation, $B=\frac{R}{R_{g} \sqrt{\xi}}$, we can change (6) into the following:

$$
\begin{aligned}
v_{\max } & =\frac{\frac{2 \gamma \cos \theta}{R}-P_{0} \frac{h_{i}}{h-h_{i}}}{2 \mu_{b} h_{i}} R^{2} S \\
S & =\left\{\frac{1}{2}+\frac{1}{2 B^{2}} \ln \left[1+B^{2}\right]-\frac{1}{B} \arctan B\right\}
\end{aligned}
$$

From the above equations (7) and (8), it can be seen that the relationship between $S$ and $B$ will reflect the influence of scale effects of the cavity on the liquid flowing velocity during forming stage.

As shown in Fig. 3, $S$ increases rapidly and starts to saturate as Bincreases to more than 40. It means that, if the characteristic length of the cavity is much larger than the radius of rotation of fluid molecular, the influence of scale effects can be neglected. Otherwise, the influence of scale effects will be very large, and the velocity of liquid flow will decrease greatly and even fall to zero.

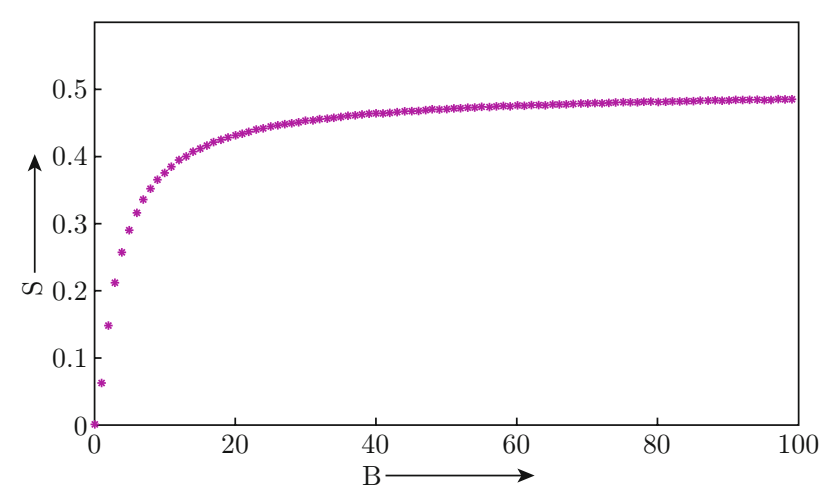

Fig. 3 Influence of scale effects of the cavity on the liquid flowing velocity.

Moreover, from the formula (5) and (6), it is obvious that: decreasing both the external air pressure and the viscosity of the fluid will increase the flowing velocity of the fluid; increasing surface tension of fluid and wettability between the liquid and cavity will also accelerate the flowing and penetrating of the liquid; and if the contact angle $\theta<90^{\circ}$, then the liquid can penetrate into the cavity.

\section{The assembly under vacuum}

As shown in Fig. 4, after the liquid cast over the cavities, the assembly is evacuated under vacuum pressure $\mathrm{P}_{v}$.

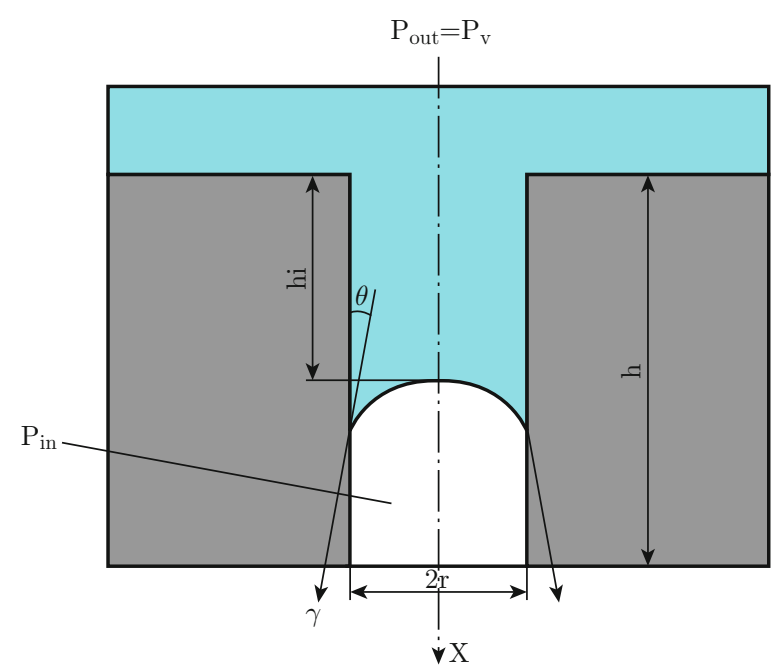

Fig. 4 The assembly vacuumized under vacuum.

Similarly, we will select a micro-cylinder in the flowing liquid, as shown in the following Fig. 5.

We can also obtain the following formula:

$$
\begin{aligned}
v= & \frac{\frac{2 \gamma \cos \theta}{R}-P_{v} \frac{h_{i}}{h-h_{i}}}{2 \mu_{b} h_{i}}\left\{\frac{1}{2}\left(R^{2}-r^{2}\right)+\frac{\xi R_{g}^{2}}{2} \ln [1+\right. \\
& \left.\left.\frac{(R-r)^{2}}{\xi R_{g}^{2}}\right]-\sqrt{\xi} R R_{g} \arctan \frac{(R-r)}{\sqrt{\xi} R_{g}}\right\}
\end{aligned}
$$




$$
\begin{aligned}
& v_{\max }= \frac{\frac{2 \gamma \cos \theta}{R}-P_{v} \frac{h_{i}}{h-h_{i}}}{2 \mu_{b} h_{i}}\left\{\frac{1}{2} R^{2}+\frac{\xi R_{g}^{2}}{2} \ln [1+\right. \\
&\left.\left.\frac{R^{2}}{\xi R_{g}^{2}}\right]-\sqrt{\xi} R R_{g} \arctan \frac{R}{\sqrt{\xi} R_{g}}\right\}
\end{aligned}
$$

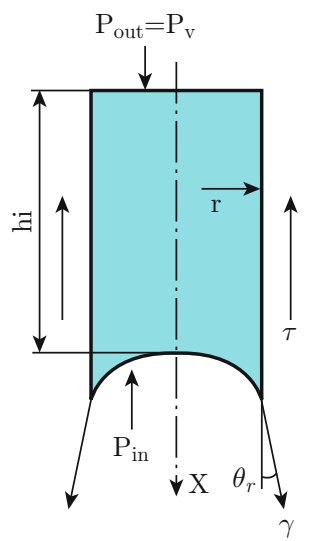

Fig. 5 State diagram of the micro cylinder under forces.

From the formula (9), it can be seen that a higher degree of vacuum will improve the flowing and penetration of liquid into the cavities. Due to the degree of vacuum can be very high, the penetration velocity of the fluid in vacuum will be a lot quicker vis-à-vis than the penetration velocity of the fluid in atmosphere.

We can estimate the maximum of penetration depth of liquid into the cavity. In the formula (10), the liquid will come to its depth limit when the $v_{\max }$ approaches zero. Therefore, we can obtain the following formula:

$$
\frac{h_{i}}{h}=\frac{1}{1+\frac{P_{v}}{2 \gamma \cos \theta / R}}
$$

As the value of $P_{v}$ can be very small, the fill ratio $\frac{h_{i}}{h}$ will be very close to $100 \%$.

The assembly is taken out to return to the atmospheric environment again

After the evacuation step, the assembly is placed in the ambient atmosphere again. As shown in Fig. 6, due to the separation by the liquid, the air pressure in the cavities is very low $\left(\mathrm{P}_{v}\right)$, while the pressure out of the cavities is much higher $\left(\mathrm{P}_{0}\right)$.

We think that, influenced by the pressure difference between the outer cavity and the inner cavity, the wettability of liquid on cavities may be changed from wetting to dewetting. Under this condition, the surface tension will contribute to drive the penetrated liquid out of the cavities.

Therefore, the time in step 3 should be shortened as soon as possible, while the liquid is better to be cured after step 2.

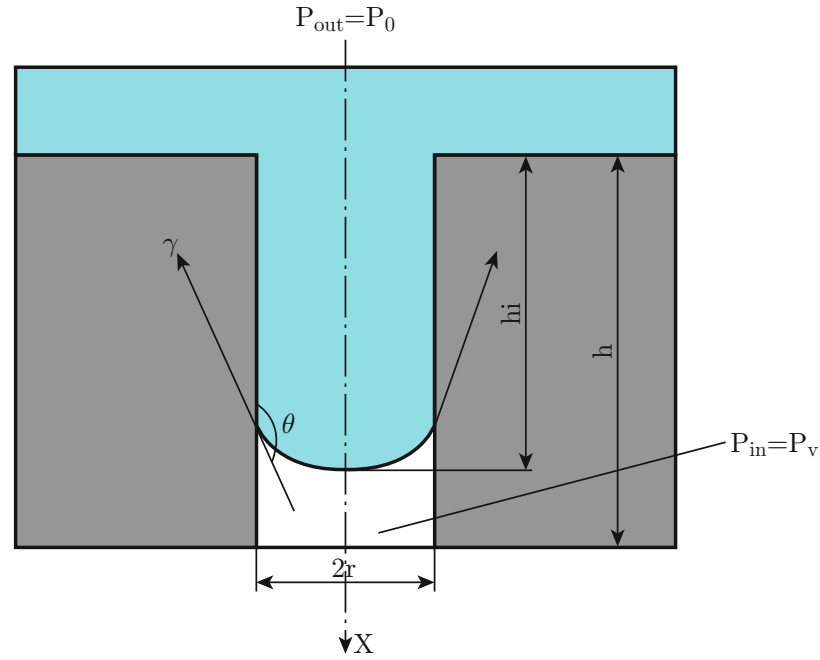

Fig. 6 The assembly is put into atmosphere again.

\section{Experiment and discussion}

In experiment, we utilize cast molding process to fabricate PDMS molds. The master template used for cast molding consists of a layer of positive resist and a silicon substrate, and the master patterns are defined in the resist by a high resolution electron beam lithography system, and the casting material is PDMS (ESSIL 291) from Axson Corporation. The whole cast molding process is similar to our previous work [17].

\section{Effects of viscosity on replication fidelity}

The viscosity or the mixed PDMS resin (ESSIL 291/ESSIL 293, weight ratio 10:1) is $40000 \mathrm{mPa} \cdot \mathrm{s}$ under room temperature. After being degassed to eliminate the air bubble, the mixture is cast over the master, and stayed for an hour. Then, the mixture is cured for 3 hours under $80^{\circ} \mathrm{C}$, and peeled off from the master under room temperature. The experimental results is shown in Fig. 7,

From the above figure, the height of features in the obtained PDMS mold is only $30 \mathrm{~nm}$, while the grooves depth in the master is $200 \mathrm{~nm}$ (width of $80 \mathrm{~nm}$ and space of $920 \mathrm{~nm}$ ). So, this reveals that the pure PDMS resin cannot penetrate into the bottom of cavity in the master due to its much higher viscosity.

However, if diluting the PDMS resin with normal heptane, we can obtain low viscosity PDMS. When the volume concentration of PDMS decreases to $30 \%$, its viscosity will decrease to $400 \mathrm{mPa} \cdot \mathrm{s}$ under room temperature. Using the diluted resin, even if the master has dense grooves with the same depth $200 \mathrm{~nm}$ (width of $80 \mathrm{~nm}$ and space of $240 \mathrm{~nm}$ ), the resin with lower viscosity can still penetrate into their bottoms, as shown in the following Fig. 8.

However, it is a pity that the cured PDMS features 


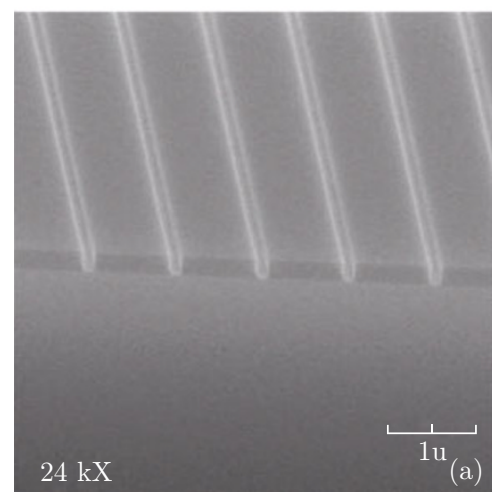

Ra:18.57 nm Rq:12.43 nm

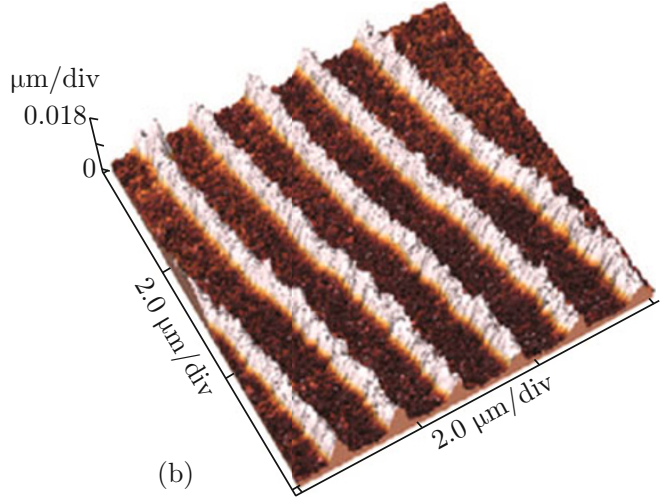

Rp:40.65 nm Rv:-11.05 nm

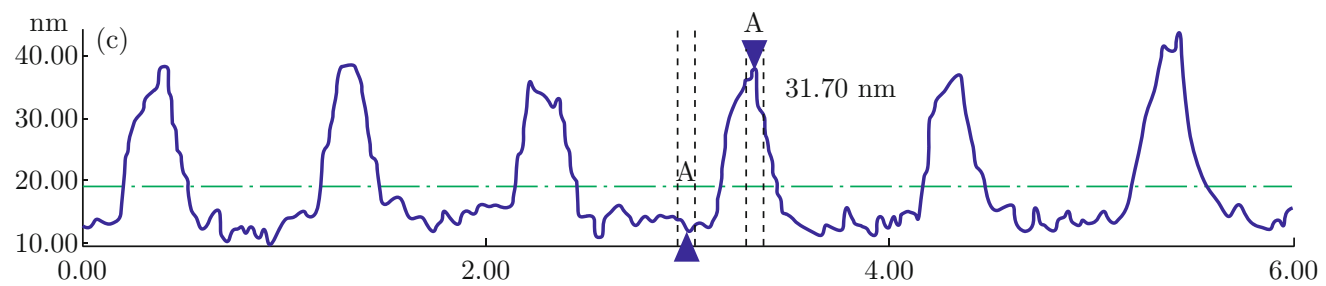

Fig. 7 Images of master and PDMS mold obtained by pure PDMS resin. (a) SEM picture of master; (b) and (c) AFM images of PDMS mold.
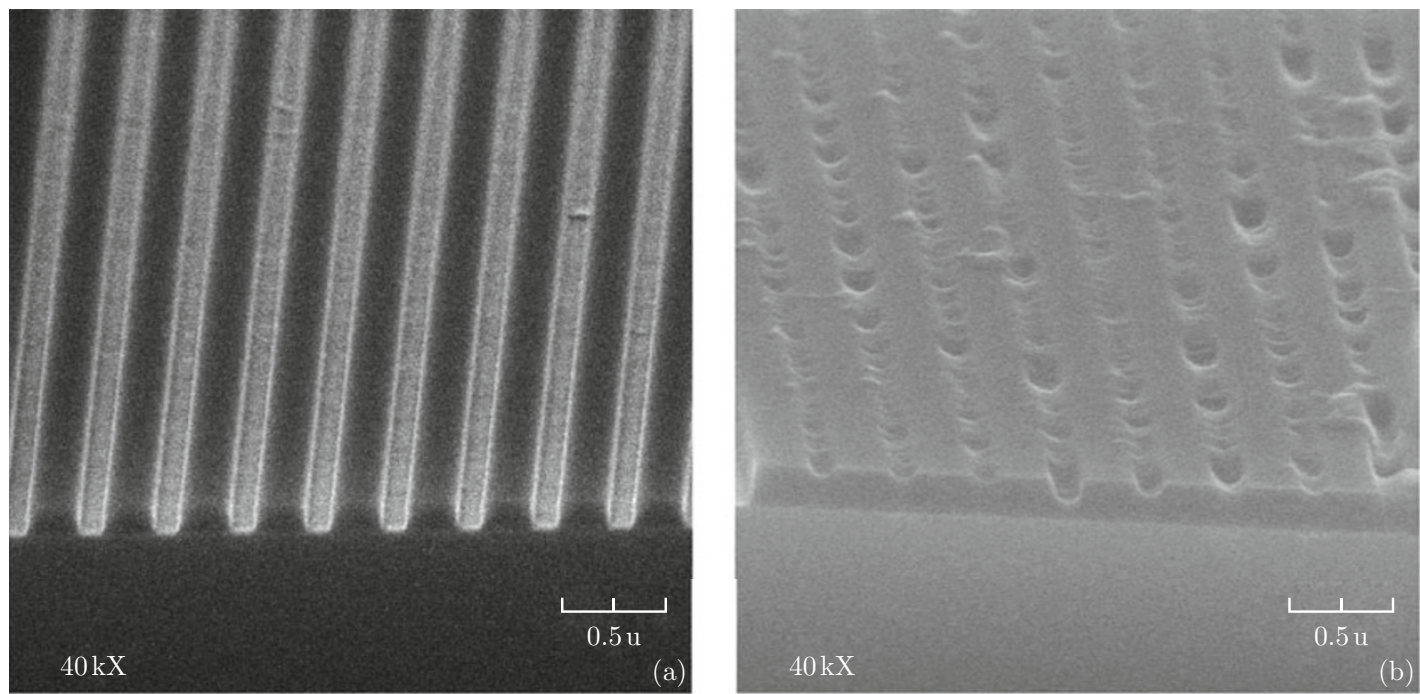

Fig. 8 Images of master and PDMS mold obtained by diluted PDMS resin. SEM picture of (a) Master; (b) Cured PDMS and master.

are almost all ruptured and trapped in the grooves of the master. The reason is not clear, but maybe due to the deterioration of the mechanical properties of PDMS after dilution, since all the other conditions during cast molding are unchanged, except for the dilution of PDMS.

\section{Effects of degree of vacuum on the replication fidelity}

In the experiment, PDMS molds are fabricated under several different pressures, such as $0.06 \mathrm{MPa}, 0.03$
MPa, $1000 \mathrm{~Pa}$, and $10 \mathrm{~Pa}$, whereas the other conditions are kept the same (curing temperature $40^{\circ} \mathrm{C}, 36$ hours). Figure 9 shows the AFM images of the final PDMS molds:

From the Fig. 9, it is obvious that, as vacuum pressure is reduced from $0.06 \mathrm{MPa}$ to $10 \mathrm{~Pa}$, the quality of the PDMS mold is improved continuously. On the one hand, feature depth in PDMS molds increases from $70 \mathrm{~nm}$ to $200 \mathrm{~nm}$. On the other hand, the defects in features are also reduced gradually. Therefore, a higher vacuum degree will be helpful to obtain a high quality replication during the cast molding process. 

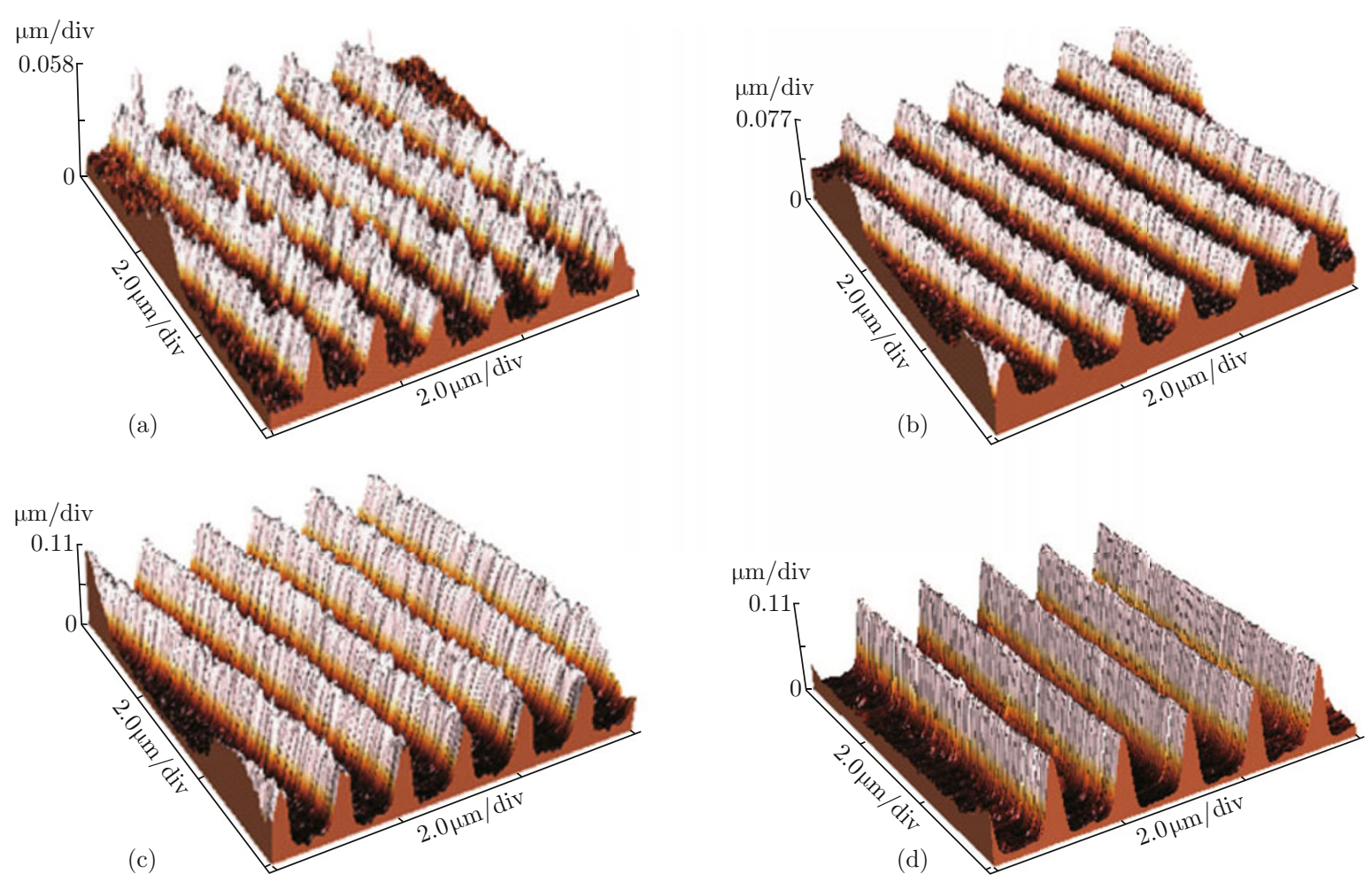

Fig. 9 AFM images of PDMS molds fabricated under different vacuum pressure. (a) Pressure of 0.06 MPa; (b) Pressure of $0.03 \mathrm{MPa}$; (c) Pressure of $1000 \mathrm{~Pa}$; (d) Pressure of $10 \mathrm{~Pa}$.

\section{Conclusions}

Through solving the flowing and filling model of liquid during the forming stage of cast molding process, factors which affect the penetrating state of the liquid have been analyzed: fluid viscosity, external air pressure, surface tension of liquid, and wettability between liquid and cavity. For the first two factors, the lower values of them will increase the flowing velocity of liquid; but for the last two factors, the effects are just the reverse. Another important factor is scale effect, which will affect the flowing velocity greatly, if the characteristic length of the cavity is comparable to the radius of rotation of liquid molecular. The experimental results also indicate that, lower viscosity and higher vacuum degree will be helpful for the liquid to penetrate into the bottom of the cavity.

\section{Acknowledgment}

The research is financially supported by NSFC under Grant No. 90923040, and by China's National "973" Program under Grant No. 2009CB724202.

\section{References}

[1] S. Masuda, M. Washizu and T. Nanba, IEEE Trans. Ind. Appl. 25, 732 (1989). http://dx.doi.org/10.

\section{$1109 / 28.31255$}

[2] B. Jo and D. J. Beebe, Proc. SPIE. 3877, 222 (1999). http://dx.doi.org/10.1117/12.359340

[3] G. Chen, G. T. Mccandless, R. L. Mccarley and S. A. Soper, Lab Chip. 7, 1424 (2007).

[4] L. Gitlin, P. Schulze and D. Belder, Lab Chip. 9, 3000 (2009). http://dx.doi.org/10.1039/b904684d

[5] L. D. Sio, J. G. Cuennet, A. E. Vasdekis and D. Psaltis, Appl. Phys. Lett. 96, 131112 (2010). http://dx.doi. org/10.1063/1.3377801

[6] Y. Xia, G. M. Whitesides, Angew. Chem. Int. Ed. Engl. 37, 550 (1998). http://dx.doi.org/ 10.1002/(SICI) 1521-3773(19980316) 37: 5<550: : AID-ANIE550>3. 0 . CO ; 2-G

[7] W. M. Choi and O. O. Park, Microelectron. Eng. 70, 131 (2003). http://dx.doi.org/10.1016/ S0167-9317 (03) 00436-2

[8] U. Plachetka, M. Bender, A. Fuchs, B. Vratzov, T. Glinsner, F. Lindner and H. Kurz, Microelectron. Eng. 73, 167 (2004). http://dx.doi.org/10.1016/ S0167-9317(04)00093-0

[9] X. Ye, Y. Ding, H. Liu and Y. Duan, Thin Solid Films 581, 6933 (2010). http://dx.doi.org/10.1016/ j.tsf. 2010.06.038

[10] X. Ye, Y. Ding, Y. Duan, H. Liu and B. Lu, J. Vac. Sci. Technol. B 28, 138 (2010). http://dx.doi.org/ 10.1116/1.3290753

[11] J. Narasimhan and I. Papautsky, J. Micromech. Microeng. 14, 96 (2004). http://dx.doi.org/10.1088/ 0960-1317/14/1/013 
[12] C. R. Martin and I. A. Aksay, J. Electroceramics. 12, 53 (2004).

[13] V. N. Goral, Y. Hsieh, O. N. Petzold, R. A. Faris and P. K. Yuen, J. Micromech. Microeng. 21, 017002 (2011). http://dx.doi.org/10.1088/0960-1317/21/ $1 / 017002$

[14] X. Ye, Y. Ding, Y. Duan, H. Liu and J. Shao, J. Vac. Sci. Technol. B 28, 86 (2010). http://dx.doi.org/10. $1116 / 1.3273601$

[15] K. Ren, W. Dai, J. Zhou, J. Su and H. Wu, PNAS 17, 8162 (2011). http://dx.doi.org/10.1073/pnas. 1100356108

[16] Y. M. Chiang, M. Bachman, C. Chu and G. P. Li,
Proc. SPIE. 3877, 303 (1999). http://dx.doi.org/ 10.1117/12.359350

[17] X. Ye, H. Liu, Y. Ding, H. Li and B. Lu, Microelectron. Eng. 86, 310 (2009). http://dx.doi.org/10.1016/j. mee. 2008.10.011

[18] S. A. Somers and H. T. Davis, J. Chem. Phys. 96, 5389 (1992). http://dx.doi.org/10.1063/1.462724

[19] K. P. Travis, B. D. Todd and D. J. Evans, Phys. Rev. E55, 4288 (1997). http://dx.doi.org/10.1103/ PhysRevE. 55.4288

[20] A. C. Eringen and K. Okada, Int. J. Eng. Sci. 33, 2297 (1995). http://dx.doi.org/10.1016/ 0020-7225 (95) 00071-5 